\title{
Outcomes of Complex Cataract Surgery in Patients with Primary Open-angle Glaucoma
}

\author{
Albert R Bargoud ${ }^{1}$, Hardik Parikh², Neil Kalbag ${ }^{3}$, Patricia Greenberg ${ }^{4}$, Albert S Khouri ${ }^{5}$
}

\begin{abstract}
Aim: Whether pupillary expansion during phacoemulsification causes a change in postoperative intraocular pressure (IOP) is currently unknown. However, a growing proportion of patients can present with concurrent glaucoma and cataracts, which poses an increased risk of having small pupils and makes finding the answer to this question imperative for treating physicians.

Materials and methods: This was a retrospective, observational cohort study which utilized data from 2008 to 2016 from the University Hospital, Newark, New Jersey, USA. All patients with primary open-angle glaucoma (POAG) who underwent phacoemulsification with pupillary expansion were considered for inclusion. Cases were subsequently excluded if they had prior incisional glaucoma surgery, if phacoemulsification was combined with another surgery, or if they had any incisional surgery in the eye 1 year preoperatively or postoperatively. The control group was made up of patients without POAG. The primary outcome was IOP.

Results: Thirty-seven eyes from 31 glaucoma patients and 29 eyes from 28 control patients met inclusion criteria. The mean IOP in the POAG group increased from $15.0 \pm 4.6 \mathrm{~mm} \mathrm{Hg}$ to $15.9 \pm 3.5 \mathrm{~mm} \mathrm{Hg}$ after 1 year, whereas the control group decreased from $14.1 \pm 3.6 \mathrm{~mm} \mathrm{Hg}$ to $11.9 \pm 3.9 \mathrm{~mm} \mathrm{Hg}$. Multivariate analysis showed that glaucoma was associated with a $5.56 \mathrm{~mm} \mathrm{Hg}$ increase in IOP at 12 months postoperatively. The average number of glaucoma medications decreased significantly from $1.7 \pm 1.4$ at the baseline to $1.3 \pm 1.3$ after 1 year.

Conclusion: In contrast with non-POAG patients, no significant drop in the mean IOP was noted after complex cataract surgery for this cohort of glaucoma patients, although medication burden significantly decreased and VA improved significantly.

Clinical significance: Phacoemulsification with intraoperative pupillary expansion in POAG patients may not decrease IOP after 12 months but it can decrease the number of anti-glaucoma medications they take.

Keywords: Cohort study, Glaucoma, Intraocular pressure, Phacoemulsification, Small pupil.

Journal of Current Glaucoma Practice (2019): 10.5005/jp-journals-10078-1250
\end{abstract}

\section{INTRODUCTION}

Glaucoma and cataracts are two of the leading causes of blindness worldwide. ${ }^{1}$ They often occur concurrently because they affect patients in the same age group. It is well documented that cataract surgery with phacoemulsification lowers IOP postoperatively, even in glaucomatous eyes. ${ }^{2-8}$ Currently, IOP is the only known modifiable risk factor for glaucoma. The mechanism that causes the IOP reduction has not been fully elucidated, but studies have shown that changes to the anterior chamber depth and angle as well as the trabecular meshwork may be responsible, among other reasons. $^{9-13}$

However, one area that has yet to be explored is whether intraoperative mechanical iris expansion affects the IOP-lowering effects of phacoemulsification in glaucoma patients. This is important because a significantly higher proportion of patients with glaucoma have been found to have smaller pupils compared with similar control groups, and they require iris manipulation and pupil expansion more frequently. ${ }^{14,15}$ Small pupils also decrease access to the lens and the size of the surgical field and are associated with multiple complications such as loss of vitreous, anterior and posterior capsule tears, and retained lens material. ${ }^{16}$ Pharmacologic agents and viscoexpansion are sometimes enough to dilate the pupil, but in some cases, a mechanical iris expansion device is used to achieve and maintain dilation throughout the procedure. Katz et al. previously showed that the decrease in IOP after combined trabeculectomy and phacoemulsification was lower in patients undergoing iris expansion when compared with a control group. ${ }^{17}$ However, to our knowledge, no paper has specifically

\footnotetext{
${ }^{1-5}$ Department of Ophthalmology, Rutgers University, Newark, New Jersey, USA
}

Corresponding Author: Albert $S$ Khouri, Department of Ophthalmology, Rutgers University, Newark, New Jersey, USA, Phone: +1 9739722065, e-mail: albert.khouri@rutgers.edu

How to cite this article: Bargoud AR, Parikh $\mathrm{H}$, et al. Outcomes of Complex Cataract Surgery in Patients with Primary Open-angle Glaucoma. J Curr Glaucoma Pract 2019;13(2):62-67.

Source of support: Nil

Conflict of interest: None

investigated how the use of intraoperative iris expansion during phacoemulsification affects the IOP in glaucoma patients.

In this study, we aim to evaluate how IOP, visual acuity (VA), and number of antiglaucoma medications are affected in patients with POAG undergoing phacoemulsification with mechanical intraoperative iris expansion. This work can help us improve management decisions regarding glaucoma patients who also develop cataracts.

\section{Materials and Methods}

A retrospective, observational chart review of complex cataract surgeries performed at the University Hospital, Newark, NJ, USA, from 2008 to 2016 was conducted. Informed consent was not required as this was a retrospective study and patient records were anonymized and de-identified prior to analysis. This study was 
conducted in accordance with the Declaration of Helsinki. Although there are multiple definitions of what characterizes a complex cataract surgery, for the purpose of this study, it was defined as a phacoemulsification with the use of intraoperative mechanical iris expansion devices (iris hooks, Kuglen hooks, and Malyugin rings). All eyes that underwent complex cataract surgery, were diagnosed with POAG, and had no incisional surgery for 1 year prior to and 1 year after phacoemulsification were considered for inclusion. Eyes were subsequently excluded if they had a prior incisional glaucoma surgery, if phacoemulsification was combined with another surgery or if the use of pupillary expansion devices was not confirmed from the operative report. Furthermore, patients in the POAG group were excluded if they did not have a confirmed glaucoma diagnosis or if they had non-POAG types of glaucoma such as neovascular, uveitic, or chronic angle closure. The control group was made up of patients without POAG, who underwent phacoemulsification with intraoperative mechanical pupillary expansion. Control patients were excluded if they had incisional surgery 1 year prior to phacoemulsification.

Eyes that developed intraoperative phacoemulsification complications that could have affected IOP outcomes (vitrectomy, anterior chamber intraocular lens (ACIOL), or sulcus placement or conversion to large incisional surgery) were also excluded from the analysis. All data were obtained from preoperative and postoperative visits as well as operative reports of the procedure. Pupils that were judged by the surgeon to be too small to allow a safe cataract extraction, generally $<5 \mathrm{~mm}$, were the indication for expansion. The use of iris hooks, Kuglen hooks, or a Malyugin ring was based on individual surgeon preference. All procedures were performed by board certified experienced surgeons. There was no significant variance in the surgical techniques of phacoemulsification and pupillary expansion among the surgeons.

The mean of two preoperative IOP measurements was used to define the preoperative baseline IOP to minimize the effects of IOP variability. When patients only had one preoperative measurement, it alone was used as a measure of baseline IOP. All patients underwent complete preoperative and postoperative examinations including VA testing, slit-lamp examination, IOP measurement with Goldmann applanation tonometry, and indirect ophthalmoscopy. Postoperative visits were scheduled for 1 day ( \pm 3 days), 1 week ( \pm 3 days), 1 month ( \pm 3 weeks), 3 months ( \pm 1 month), 6 months ( \pm 2 months), and 1 year ( \pm 4 months). The primary outcome was IOP and the secondary outcomes were the number of glaucoma medications, VA, survival analysis for decreased IOP, and intraoperative and postoperative complications. When counting the number of glaucoma medications, combination medications such as Cosopt, Simbrinza, and Combigan were counted as two medications.

\section{Statistical Methods}

Summary statistics including mean and standard deviation as well as frequency and percentage were used to first describe the two patient groups. To compare the stated outcomes between the control and POAG groups, an independent two-sample $t$ test was used. When comparing values from preoperative measurements vs different follow-up time points for the same patient, a paired $t$ test was used. Further, to control for the possibility that multiple eyes being contributed from the same patient are not necessarily independent when considering the study outcomes, we also performed an independent two sample $t$ test in which we averaged the IOP measurements in patients with two eyes included in the study as described by Huang et al. ${ }^{18}$ We also performed multivariate analyses using a residual maximum likelihood (REML) mixed effects model (MEM) to assess the effect of the following variables on IOP: presence of glaucoma, age, sex, cataract density (mature vs visually significant), eye operated on (right vs left), number of preoperative antiglaucoma medications taken, and type of intraoperative pupillary expansion device used (iris hook vs Kuglen hook vs Malyugin ring). A $p$ value less than 0.05 was considered statistically significant.

Additionally, a post hoc power analysis was done, which revealed that a total sample size of 25 eyes was the minimum number of eyes needed to detect a statistically significant change in IOP with $80 \%$ statistical power and an alpha error of 0.05 . Continuous measures are denoted as mean \pm standard deviation. Statistical analysis was performed using the R: a language and environment for statistical computing (R Core Team (2018). R: a language and environment for statistical computing. R Foundation for Statistical Computing, Vienna, Austria; URL https://www.R-project.org/), GraphPad Prism 8.1.1 (San Diego, CA, USA) and MedCalc Statistical Software version 18.11.3 (MedCalc Software bvba, Ostend, Belgium; https://www. medcalc.org; 2019).

\section{Results}

Baseline characteristics of the POAG ( $n=37$ eyes) and control $(n=29$ eyes) groups are shown in Table 1. The POAG group was significantly older than the control group at the time of surgery $(72.5 \pm 10.2 \mathrm{vs}$ $65.3 \pm 11.5$ years old; $p=0.01$ ). However, there were no significant differences in the proportions of hypertension, diabetes, or hyperlipidemia between the control and the POAG group. Of note, one patient in the POAG group had Fuchs endothelial dystrophy. The number of eyes at preoperative and postoperative visits is shown in Table 2.

First, we analyzed the change in IOP after complex cataract surgery in both groups (Fig. 1). Significant differences in the control group at the postoperative visits compared to preoperative are denoted with "a" (paired Students $t$ test). Significant differences

Table 1: Baseline characteristics of the POAG and control groups

\begin{tabular}{lll}
\hline Variable & Control & POAG \\
\hline $\begin{array}{l}\text { Number of eyes (number of } \\
\text { patients) }\end{array}$ & $29(28)$ & $37(31)$ \\
$\begin{array}{l}\text { Age (mean } \pm \text { SD) } \\
\text { Eyes in female patients }\end{array}$ & $65.3 \pm 11.5$ & $72.5 \pm 10.2^{*}$ \\
$\begin{array}{l}\text { Follow up, days (mean } \pm \text { SD) } \\
\text { Past medical history }\end{array}$ & $465.4 \pm 411.8$ & $708.6 \pm 561.4$ \\
Hypertension & $19 / 29(65.5 \%)$ & $25 / 37(67.6 \%)$ \\
$\begin{array}{l}\text { Type Il diabetes } \\
\text { Hyperlipidemia }\end{array}$ & $13 / 29(44.8 \%)$ & $10 / 37(27.0 \%)$ \\
$\begin{array}{l}\text { Ocular characteristics } \\
\text { Mature cataract }\end{array}$ & $9 / 29(31.0 \%)$ & $11 / 37(29.7 \%)$ \\
Kuglen hook & $3 / 29(10.3 \%)$ & $5 / 37(13.5 \%)$ \\
lris hook & $1 / 29(3.4 \%)$ & $7 / 37(18.9 \%)$ \\
Malyugin ring & $22 / 29(75.9 \%)$ & $28 / 37(75.7 \%)$ \\
\hline
\end{tabular}

${ }^{*} p=0.012$ on independent Students $t$ test (no other variables showed a significant difference)

SD, standard deviation 
Table 2: Number of eyes at each visit in the POAG and control groups

\begin{tabular}{lll}
\hline Time point & Control & POAG \\
\hline Preoperative & 29 & 37 \\
& $(100.0 \%)$ & $(100.0 \%)$ \\
1 Day & $25(86.2 \%)$ & $30(81.1 \%)$ \\
1 Week & $24(82.8 \%)$ & $28(75.7 \%)$ \\
1 Month & $19(65.5 \%)$ & $34(91.9 \%)$ \\
3 Months & $15(51.7 \%)$ & $28(75.7 \%)$ \\
6 Months & $14(48.3 \%)$ & $25(67.6 \%)$ \\
12 Months & $18(62.1 \%)$ & $28(75.7 \%)$ \\
\hline
\end{tabular}

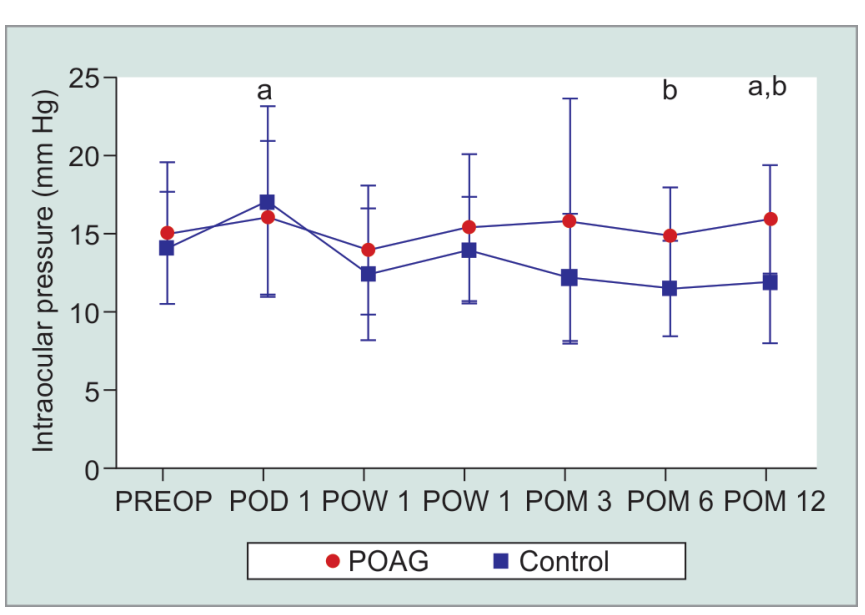

Fig. 1: Mean IOP in the POAG and control groups

in IOP between the two groups at each time point are marked with " $b$ " (independent Students $t$ test). The control and the POAG group were analyzed by $t$ tests as shown in Table 3. One model assumed that eyes were independent of each other when a patient had two eyes enrolled in the same group and the second model averaged the IOP when two eyes of the same patient were in the same group to account for possible dependence. The POAG group had an increase in IOP from $15.0 \pm 4.6$ to $15.9 \pm 3.5$, whereas the control experienced a decrease in IOP from $14.1 \pm 3.6$ to $11.9 \pm 3.9$ at 12 months postoperative. In both models, IOP between the

Table 3: Analysis of IOP in the POAG and control groups assuming independence and dependence when two eyes of the same patient were in the same group

\begin{tabular}{|c|c|c|c|}
\hline & Control & POAG & $\begin{array}{l}\text { p value for } \\
12 \text { months vs } \\
\text { preoperative } \\
\text { (by group) }^{a}\end{array}$ \\
\hline \multicolumn{4}{|c|}{ Method 1: assumes eyes are independent } \\
\hline Preoperative IOP & $14.1 \pm 3.6$ & $15.0 \pm 4.6$ & 0.501 \\
\hline 12 month post-operative IOP & $11.9 \pm 3.9$ & $15.9 \pm 3.5$ & 0.001 \\
\hline $\begin{array}{l}p \text { value for } 12 \text { months vs } \\
\text { preoperative IOP (by eye) }\end{array}$ & 0.052 & 0.012 & \\
\hline \multicolumn{4}{|c|}{$\begin{array}{l}\text { Method 2: assumes eyes are not independent average IOP for both } \\
\text { eyes) }\end{array}$} \\
\hline Preoperative IOP & $14.1 \pm 3.8$ & $14.9 \pm 4.8$ & 0.509 \\
\hline 12 month postoperative IOP & $12.0 \pm 4.0$ & $16.0 \pm 3.7$ & 0.003 \\
\hline $\begin{array}{l}p \text { value for } 12 \text { months vs } \\
\text { preoperative IOP (by eye) }\end{array}$ & 0.166 & 0.023 & \\
\hline
\end{tabular}

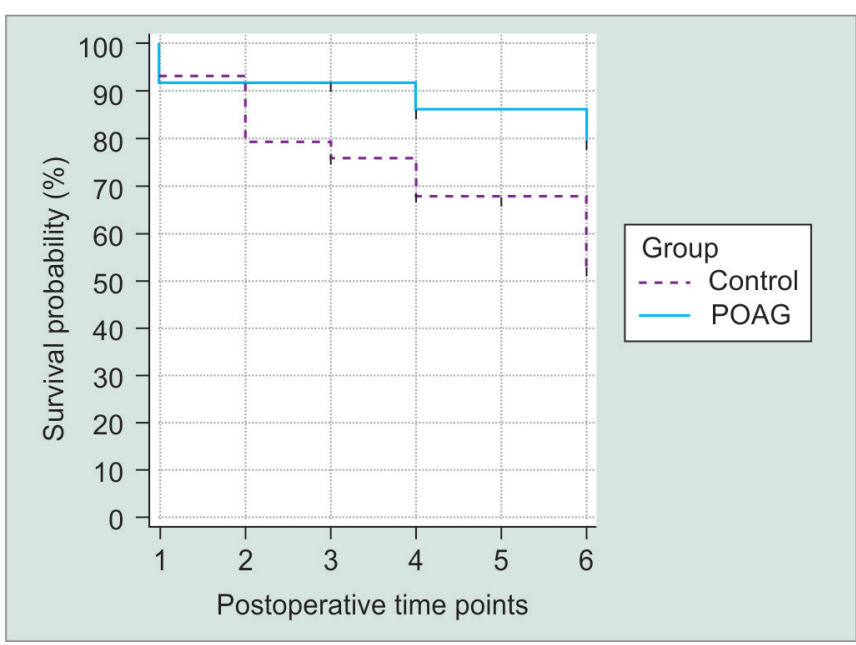

Fig. 2: Probability of survival for eyes with a $>20 \%$ decrease in IOP

glaucoma and the control group was similar at the baseline and after 3,6, and 12 months, the control group had a significantly lower IOP (all $p<0.05$ except at 3 months when eyes were assumed to be independent of each other $p=0.05$ ). Both models also show that in the POAG group, no time point had a significantly different IOP when compared to preoperative values using a paired $t$ test. Conversely, both models show the IOP in the control group to be significantly lower at 12 months when compared to preoperative values.

Additionally, a comparison of the probability of survival was performed for patients in both groups who experienced at least a $20 \%$ decrease in IOP from baseline using Kaplan-Meier methods (Fig. 2). At the 12-month time point, the cumulative probability for survival in the control and POAG groups was $58.6 \%$ and $81.0 \%$, respectively. A comparison of the two groups with a log-rank test showed a significant difference between the two curves $(p=0.03)$. Next, we ran a MEM to assess the effect of select variables on average IOP of the cohort (Table 4). This model showed that glaucoma was associated with a $5.56 \mathrm{~mm} \mathrm{Hg}$ increase in IOP at 12 months postoperatively $(p=0.005)$. None of the other variables were found to be associated with a change in IOP.

The POAG group showed a significant decrease in mean antiglaucoma medication burden at all postoperative time points except for 6 months (Table 5). The mean preoperative number of glaucoma medications was $1.7 \pm 1.4$ compared to $1.3 \pm 1.3$ at 12 months postoperatively, which represented a $24 \%$ mean overall

Table 4: Multivariate analysis of variables affecting IOP using a mixed effects model

\begin{tabular}{lrll}
\hline & \multicolumn{1}{l}{$\beta$} & REML SE (naive) & $p$ value \\
\hline Right eye & -1.84 & 0.96 & 0.30 \\
Presence of glaucoma & 5.56 & 1.86 & 0.01 \\
Age (years) & -0.09 & 0.05 & 0.34 \\
Female & 1.87 & 1.27 & 0.38 \\
Types of cataract & -0.96 & 1.42 & 0.62 \\
Number of preoperative & -0.50 & 0.62 & 0.57 \\
glaucoma medications & & & \\
Use of a Iris hook & 3.47 & 5.44 & 0.53 \\
Use of a Malyugin ring & 0.89 & 3.81 & 0.82 \\
Use of a Kuglen hook & 3.85 & 5.83 & 0.51 \\
\hline
\end{tabular}

$\mathrm{SE}$, standard error 
Table 5: Mean number of antiglaucoma medications taken by eyes in the POAG group

\begin{tabular}{lllllll}
\hline & Preop & POW 1 & POM 1 & POM 3 & POM 6 & POM 12 \\
\hline $\begin{array}{l}\text { Number of antiglaucoma } \\
\text { medications (mean } \pm \text { SD) }\end{array}$ & $1.7 \pm 1.4$ & $10 \pm 12$ & $1.0 \pm 1.2$ & $1.2 \pm 1.3$ & $1.6 \pm 1.6$ & $1.3 \pm 1.3$ \\
$p$ value (paired Students $t$ test) & & $<0.01$ & $<0.01$ & 0.02 & 0.30 & 0.02 \\
\hline
\end{tabular}

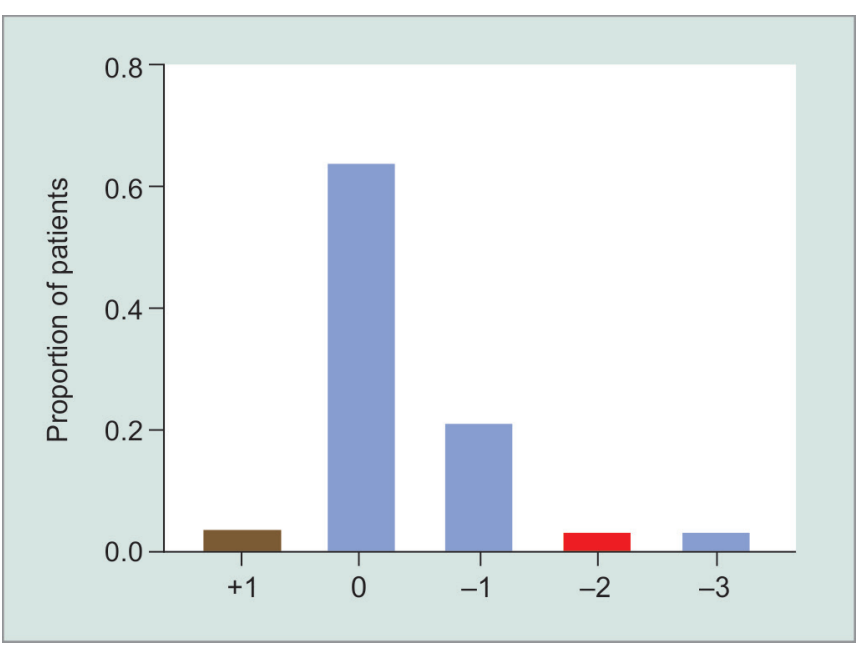

Fig. 3: Change in the number of antiglaucoma medications at 1 year compared to preoperatively

reduction in medications. At 12 months postoperatively, eight eyes in the POAG group required less antiglaucoma medications compared to the baseline (Fig. 3).

Furthermore, the control and POAG groups experienced a significant improvement in VA after 12 months. The control group logMAR decreased from $1.73 \pm 0.79$ to $0.86 \pm 0.96$ (paired $t$ test $p<0.004$ ) and the POAG group decreased from $0.88 \pm 0.58$ to $0.63 \pm 0.56$ (paired $t$ test $p<0.002$ ) after 12 months. VA was significantly improved at all time points in both groups (except for on POD1 in the POAG group $p=0.63$ ) when compared to preoperative values using a paired $t$ test. The POAG group had better final VA after 12 months, but the control group experienced a larger improvement (50.6\% vs $28.1 \%$ in the POAG group). Of note, the control group had three (10.3\%) eyes with mature cataracts and the POAG group had five (13.5\%), while all the other eyes in both groups had non-mature cataracts.

Intraoperative characteristics and postoperative complications are shown in Table 6. None of the listed variables were statistically different between the control and the POAG group. We found that of the patients with postoperative day 1 (POD1) IOP recorded, $6 / 30$ (20.0\%) in the POAG group and 5/25 (20\%) in the control group had an IOP $\geq 21 \mathrm{~mm} \mathrm{Hg}$. In all cases, the pressure decreased to $<21 \mathrm{~mm}$ $\mathrm{Hg}$ at the subsequent visit. The preoperative IOP in patients who had an IOP $\geq 21 \mathrm{~mm} \mathrm{Hg}$ on POD1 was higher when compared to patients with no spike in the glaucoma (spike: $16.3 \pm 3.5$ vs no spike: $14.8 \pm 4.7 ; p=0.45$ ) or the control group (spike: $15.6 \pm 3.5$ vs no spike: $13.8 \pm 2.5 ; p=0.31$ ); however, these changes were not significant.

\section{Discussion}

Patients with POAG who underwent complex cataract surgery did not have a reduction in IOP at any postoperative time point and had a $6 \%$ increase in IOP at 12 months, whereas patients without glaucoma experienced a $16 \%$ decrease in their IOP after 1 year. However, glaucoma patients did have a $24 \%$ decrease in mean medication burden and significantly improved VA after 1 year. Multivariate analyses showed that the presence of glaucoma was associated with a significant $5.56 \mathrm{~mm} \mathrm{Hg}$ increase in IOP compared to cases without glaucoma. Kaplan-Meier analysis shows that the presence of glaucoma significantly affected the proportion of patients who experienced $a \geq 20 \%$ decrease in IOP. All in all, these results suggest that glaucoma prevents a decrease in IOP after complex cataract surgery in this cohort.

There are no studies that specifically examined POAG patients undergoing phacoemulsification with mechanical iris expansion, but there is literature on POAG patients undergoing phacoemulsification. Chen et al. compared nine studies that examined the effect of phacoemulsification on IOP in POAG patients. ${ }^{19}$ Two of these studies were prospective and six studies included normal-tension glaucoma. There were a total of 461 POAG patients examined and the number of patients requiring mechanical iris expansion was not specified. When these studies were combined, the mean preoperative IOP was $17.7 \pm 2.0$ which decreased to $15.4 \pm 2.0$ after a mean of $16.7 \pm 6.8$ months. They also found a reduction in antiglaucoma medications from $1.7 \pm 0.4$ to $1.5 \pm 0.6$ ( $12 \%$ decrease). Given these findings, it is possible that the addition of intraoperative mechanical iris expansion in

Table 6: Intraoperative characteristics and postoperative complications of complex cataract surgery in POAG and control eyes

\begin{tabular}{llllll}
\hline Variable & Control & POAG & OR & $95 \% \mathrm{Cl}$ & $p$ value \\
\hline Intraoperative characteristics & & & & & \\
Capsular staining & $21(72.4 \%)$ & $23(62.2 \%)$ & 0.63 & $0.22-1.79$ & 0.38 \\
Synechialysis & $310.3 \%)$ & $7(18.9 \%)$ & 2.02 & $0.47-8.63$ & 0.34 \\
Suture placement & $10(34.5 \%)$ & $8(21.6 \%)$ & 0.52 & $0.18-1.57$ & 0.25 \\
Pupilloplasty & $0(0 \%)$ & $3(8.1 \%)$ & 5.99 & $0.30-120.67$ & 0.24 \\
Postoperative complications & & & & & 1.00 \\
IOP $>21$ on POD1 & & & & & 0.59 \\
Wound leak & $5(\%)$ & $1(2.7 \%)$ & 2.42 & $0.10-61.74$ & 0.71 \\
Cystoid macular edema & $0(0 \%)$ & $2(5.4 \%)$ & 1.60 & $0.14-18.57$ & \\
\hline
\end{tabular}

$\mathrm{OR}$, odds ratio; $\mathrm{Cl}$, confidence interval; $\mathrm{POD} 1$, postoperative day 1

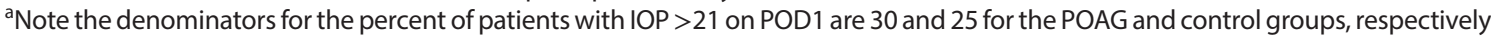


our study prevented POAG patients from experiencing a similar decrease in IOP.

One possible explanation is that the added inflammation caused by pupillary expansion exceeded the capacity of the IOPlowering mechanisms of phacoemulsification in POAG patients. Tan et al. created a model of the iris and performed a finite element analysis (FEA) to compare the stress that various pupillary expansion devices had on the iris compared to uniform circular expansion, which mimics physiologic iris expansion. ${ }^{20} \mathrm{~A}$ FEA is a tool commonly used in engineering, in which they divide the structure into smaller, finite parts, which make it possible to perform a structural analysis with a defined stress. They found that pupillary expansion devices exerted a significantly higher stress on the iris when compared to uniform circular expansion. The stress from the pupil expansion devices was the greatest at the point of contact, the pupillary margin, and decreased as it traveled toward the limbus. Stress gradients, and particularly the rise time, have been shown to induce tissue damage. ${ }^{21}$ Conversely, phacoemulsification was shown to increase the expression of interleukin-1, which increases aqueous outflow in the eye by restructuring the trabecular meshwork through the synthesis of matrix metalloproteinases. ${ }^{22,23}$ It has been proposed that IL-1 can be protective in the short run but can be damaging when chronically increased. ${ }^{13,22}$ Glaucoma patients were found to have constitutively increased levels of IL-1 in response to the increased IOP and when these patients underwent phacoemulsification, IL-1 levels increase even more. ${ }^{22}$ Therefore, it is possible that the combination of preexisting POAG and the increased stress induced by iris expansion supersedes the IOP lowering effects of phacoemulsification in this cohort. More work needs to be done in this area to evaluate how intraoperative pupillary expansion affects IOP clinically and on a molecular level, especially in glaucoma patients.

Next, we found that VA improved significantly in both the control and the POAG group when compared to the baseline. This was expected and is in line with existing literature. ${ }^{15}$ Although the POAG group had a better final VA, the control group experienced a greater improvement after 12 months. Undergoing cataract surgery allows for clearer optical media and improved VA. As mentioned in other studies, obtaining a new VA baseline and the ability to obtain better quality glaucoma diagnostic structural tests after surgery will allow for improved follow up over time. ${ }^{24}$

Another important consideration when weighing the risks of surgery are postoperative complications, we did not find any significant difference between the POAG and control group in terms of POD1 IOP spike, wound leaks, or cystoid macular edema. Of note, we did not have any patients in either group experience retinal detachment or endophthalmitis. For the IOP spike, there is no exact agreed upon rise in IOP that defines what constitutes an IOP spike. Some studies have used relative values whereas others have defined it using absolute thresholds. This is an important topic to address because IOP spikes may worsen glaucoma; however, the extent of this in the literature is not entirely ellucidated. ${ }^{25,26}$ The POAG group had a mean increase of $6.7 \%$ from the preoperative IOP on POD1, compared to $21.3 \%$ in the control group. This could be explained by the fact that POAG patients were taking antiglaucoma medications to lower IOP, whereas the control group were not. Chen et al. found that among six studies, the IOP spike on POD1 ranges between $3 \%$ and $27 \%$ (median $17.5 \%$ ), which is consistent with our findings. ${ }^{19}$ Given the different definitions of IOP spikes in those studies and the fact that not all of them report the proportion of cataract surgery with iris expansion, it is difficult to assess the effect of intraoperative iris expanders on IOP in the literature. Slabbagh et al. found that of 47 patients who had iris manipulation with Kuglen hooks, 12 (26\%) had an IOP spike. They defined an IOP spike as a percentage change from baseline IOP. Moreover, Turalba et al. conducted a large retrospective cohort study in which they examined 608 glaucoma patients and 4,306 control patients undergoing a planned cataract surgery and reported surgical outcomes. ${ }^{15}$ They found that $3.5 \%$ of glaucoma patients developed cystoid macular edema (CME) compared to $2.2 \%$ in the control group (OR $1.36 p=0.28$ ). Shingleton et al. found that $1.4 \%$ patients with severe glaucoma had cystoid macular edema (OR not available). ${ }^{5}$ Our results show slightly higher proportion of CME in glaucoma patients compared to controls (5.4\% vs $3.4 \%$, respectively; OR $1.60 p=0.71$ ) and to the available literature. A larger study is needed to validate these findings.

There were several limitations to this study that should be acknowledged. First, are the inherent limitations to this being a retrospective analysis with an expected loss to follow up. For a clinically relevant analysis we aimed to collect data for up to 12 months but $24.3 \%$ of patients were lost to follow up at that point, which is not atypical for these types of studies. Moreover, there was no randomization or investigator masking. In some cases, there was only one preoperative IOP reading, which may have introduced more variability into the baseline readings and the subsequent analysis causing regression to the mean, which has been previously discussed in the literature. ${ }^{8}$ An inherent limitation to any glaucoma study is the potential nonadherence to antiglaucoma therapy and the effects that may have on IOP. Effects of medication nonadherence on IOP may have influenced the POAG group, ${ }^{27}$ so it is difficult to determine what changes were due to iris expansion during phacoemulsification and what were due to medication nonadherence. Furthermore, postoperative steroids may have induced IOP elevation in both groups; however they were given to both groups. Lastly, this is a single site study performed at a referral hospital-based practice so we may have included patients with more severe pathology in both groups.

\section{Conclusion}

Complex cataract surgery in POAG patients did not decrease IOP at 12 months postoperatively in this cohort. However, POAG patients had significantly improved VA and a $24 \%$ mean overall reduction in medication burden 1 year after surgery. Multivariate and Kaplan-Meier analyzes show that glaucoma was associated with an increase in IOP. This work can be used to improve treatment protocols for patients with glaucoma and cataracts, but more work should be done to investigate how mechanical iris expansion during phacoemulsification affects IOP and to determine which patients are at risk for poor intraoperative mydriasis.

\section{Clinical Significance}

Phacoemulsification with intraoperative pupillary expansion in POAG patients may not decrease IOP after 12 months but it can decrease the number of glaucoma medications they take.

\section{References}

1. Pascolini D, Mariotti SP. Global estimates of visual impairment: 2010. Br J Ophthalmol 2012;96(5):614-618. DOI: 10.1136/ bjophthalmol-2011-300539. 
2. Obstbaum SA. Glaucoma and intraocular lens implantation. J Cataract Refract Surg 1986;12(3):257-261.

3. Perasalo R. Phaco-emulsification of cataract in eyes with glaucoma. Acta Ophthalmol Scand 1997;75(3):299-300.

4. Kim DD, Doyle JW, et al. Intraocular pressure reduction following phacoemulsification cataract extraction with posterior chamber lens implantation in glaucoma patients. Ophthalmic Surg Lasers 1999;30(1):37-40.

5. Shingleton BJ, Gamell LS, et al. Long-term changes in intraocular pressure after clear corneal phacoemulsification: normal patients vs glaucoma suspect and glaucoma patients. J Cataract Refract Surg 1999;25(7):885-890.

6. Mierzejewski A, Eliks I, et al. Cataract phacoemulsification and intraocular pressure in glaucoma patients. Klin Oczna 2008;110(1-3): 11-17.

7. Mathalone N, Hyams $\mathrm{M}$, et al. Long-term intraocular pressure control after clear corneal phacoemulsification in glaucoma patients. J Cataract Refract Surg 2005;31(3):479-483. DOI: 10.1016/ j.jcrs.2004.06.046.

8. Shrivastava A, Singh K. The effect of cataract extraction on intraocular pressure. Curr Opin Ophthalmol 2010;21(2):118-122. DOI: 10.1097/ ICU.0b013e3283360ac3.

9. Hayashi K, Hayashi $\mathrm{H}$, et al. Changes in anterior chamber angle width and depth after intraocular lens implantation in eyes with glaucoma. Ophthalmology 2000;107(4):698-703. DOI: 10.1016/s01616420(00)00007-5.

10. Dooley I, Charalampidou S, et al. Changes in intraocular pressure and anterior segment morphometry after uneventful phacoemulsification cataract surgery. Eye (Lond) 2010;24(4):519-526; quiz 527\#\#10.1038/ eye.2009.339.

11. Alaghband $\mathrm{P}$, Beltran-Agullo $\mathrm{L}$, et al. Effect of phacoemulsification on facility of outflow. Br J Ophthalmol 2018;102(11):1520-1526.

12. Meyer MA, Savitt ML, et al. The effect of phacoemulsification on aqueous outflow facility. Ophthalmology 1997;104(8):1221-1227. DOI: 10.1016/s0161-6420(97)30154-7.

13. Wang N, Chintala SK, et al. Activation of a tissue-specific stress response in the aqueous outflow pathway of the eye defines the glaucoma disease phenotype. Nat Med 2001;7(3):304-309. DOI: 10.1038/85446.

14. Park JW, Kang BH, et al. Analysis of various factors affecting pupil size in patients with glaucoma. BMC Ophthalmol 2017;17(1):168. DOI: 10.1186/s12886-017-0564-6.
15. Turalba A, Payal AR, et al. Cataract Surgery Outcomes in Glaucomatous Eyes: Results From the Veterans Affairs Ophthalmic Surgery Outcomes Data Project. Am J Ophthalmol 2015;160(4):693-701 e691. DOI: 10.1016/j.ajo.2015.07.020.

16. Malyugin B. Cataract surgery in small pupils. Indian J Ophthalmol 2017;65(12):1323-1328. DOI: 10.4103/ijo.IJO_800_17.

17. Katz LJ, Zangalli C, et al. Combined cataract and glaucoma surgery: the effect of pupil enlargement on surgical outcomes (an American Ophthalmological Society thesis). Trans Am Ophthalmol Soc 2013;111:155-168.

18. Huang J, Huang J, et al. Evaluation of Approaches to Analyzing Continuous Correlated Eye Data When SampleSize Is Small.Ophthalmic Epidemiol 2018;25(1):45-54. DOI: 10.1080/09286586.2017.1339809.

19. Chen PP, Lin SC, et al. The Effect of Phacoemulsification on Intraocular Pressure in Glaucoma Patients: A Report by the American Academy of Ophthalmology. Ophthalmology 2015;122(7):1294-1307. DOI: 10.1016/j.ophtha.2015.03.021.

20. Tan RKY, Wang $X$, et al. Numerical stress analysis of the iris tissue induced by pupil expansion: Comparison of commercial devices. PLoS One 2018;13(3):e0194141. DOI: 10.1371/journal.pone.0194141.

21. Doukas AG, McAuliffe DJ, et al. Physical factors involved in stresswave-induced cell injury: the effect of stress gradient. Ultrasound Med Biol 1995;21(7):961-967.

22. Wang N, Chintala SK, et al. Ultrasound activates the TM ELAM-1/ IL-1/NF-kappaB response: a potential mechanism for intraocular pressure reduction after phacoemulsification. Invest Ophthalmol Vis Sci 2003;44(5):1977-1981. DOI: 10.1167/iovs.02-0631.

23. Kee $C$, Seo K. The effect of interleukin-1alpha on outflow facility in rat eyes. J Glaucoma 1997;6(4):246-249.

24. Kung JS, Choi DY, et al. Cataract surgery in the glaucoma patient Middle East Afr J Ophthalmol 2015;22(1):10-17. DOI: 10.4103/09749233.148343

25. Chen PP, Musch DC, et al. The effect of early posttrabeculectomy intraocular pressure spike in the collaborative initial glaucoma treatment study. J Glaucoma 2011;20(4):211-214. DOI: 10.1097/ IJG.0b013e3181e07947.

26. Aung T, Oen FT, et al. Randomised controlled trial comparing the effect of brimonidine and timolol on visual field loss after acute primary angle closure. Br J Ophthalmol 2004;88(1):88-94. DOI: 10.1136/bjo.88.1.88.

27. Tsai JC. A comprehensive perspective on patient adherence to topical glaucoma therapy. Ophthalmology 2009;116(11 Suppl):S30-S36. DOI: 10.1016/j.ophtha.2009.06.024. 